\title{
Dynamic Aperture Evaluation at the current working point for RHIC polarized proton run
}

\author{
Y. Luo, M. Bai, J. Beebe-Wang, R. Calaga, W. Fischer, \\ C. Montag, S. Tepikian, D. Trbojevic \\ Brookhaven National Laboratory
}

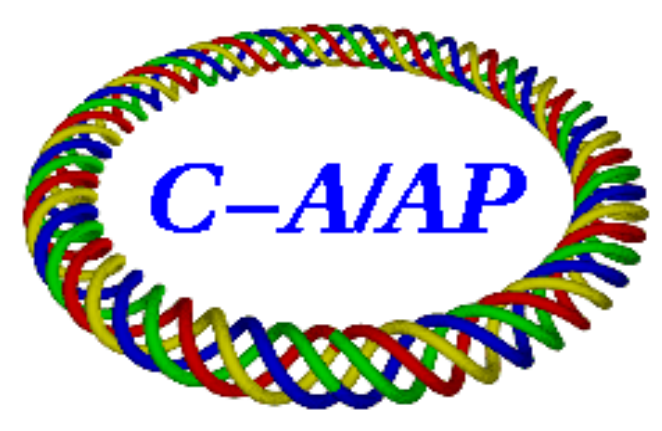

Collider-Accelerator Department Brookhaven National Laboratory Upton, NY 11973 
$\mathrm{CAD} / \mathrm{AP} / 271$

April 5, 2007

\title{
Dynamic Aperture evaluation at the current working point for RHIC polarized proton run
}

\author{
Y. Luo, M. Bai, J. Beebe-Wang, R. Calaga, W. Fischer, \\ C. Montag, S. Tepikian, D. Trbojevic \\ Brookhaven National Laboratory, Upton, NY 11973, USA
}

With the updated multipole magnet field errors in the interaction regions (IRs), detailed dynamic aperture studies are carried out around the current RHIC polarized proton (pp) working point. The beam parameters and $\beta^{*}$ s are similar to those proposed for the next pp run. The effects on the dynamic apertures from nonlinear corrections, such as multipole field error correction in the IRs, second order chromaticity correction and horizontal third order resonance correction are evaluated. The sextupole components in the arc dipoles and the observed tune ripples are also considered.

\section{Introduction}

At the current working point for the RHIC polarization proton (pp) run, the fractional tunes are constraint between $2 / 3$ and 0.7 . The vertical faction tune 0.7 will impact both the luminosity lifetime and the polarization. And when the horizontal tune is close to $2 / 3$, the beam lifetime is affected by the third order betatron resonance.

To further increase the luminosity, we can increase the bunch intensity $N_{b}$ and reduce the $\beta^{*}$. At 100 $\mathrm{GeV}$, assuming $N_{b}=2.0 \times 10^{11}, \beta^{*}=0.9 \mathrm{~m}$ at two collision points IP6 and IP8 and and the normalized transverse rms emittance $\epsilon_{r m s, n o r m}=2.5 \times 10^{-6} \mathrm{~m} . \mathrm{rad}$, the total beam-beam tune shift is 0.02 . Therefore, the non-collisional horizontal tune should be larger than $0.666+0.02=0.686$. According to Ref. [1], to increase the tune space to accommodate the beam-beam tune spread and to get higher luminosity, both nonlinear chromaticities and horizontal third order resonance have to be corrected.

There are a total of 144 sextupole magnets in the 6 arcs of each RHIC ring. In previous RHIC pp runs, they were sorted into two families, one focusing and one defocussing, which allowed only first order chromaticity correction. In the $2007 \mathrm{Au}$ run, the number of arc sextupole power supplies were doubled from 12 to 24 . S. Tepikian proposed a 8-family scheme to compensate the second and third order chromaticities [2]. In the scheme, each outer or inner arc has 4 sextupole families, and all outer or inner arcs have the same sextupole strength patterns. An online nonlinear chromaticity correction based on off-momentum tune response matrix was proposed in Ref. [3].

In the 2006 RHIC pp run, the correction of the horizontal third order resonance $3 Q_{x}$ was tested at injection, where 12 sextupole families were used [4, 7]. The key point for this correction scheme is to measure the driving term $h_{30000}$ of the $3 Q_{x}$ resonance. The technique of measuring $h_{30000}$ with an AC dipole is still under evaluation [5]. In Ref. [6], 12 local sextupole correctors in the interaction regions (IRs) are suggested for the $3 Q_{x}$ resonance correction. The merit of using IR sextupole correctors is to separate the nonlinear chromaticity correction and third order resonance $3 Q_{x}$ correction. In the following, we will use the arc sextupole families for the second order chromaticity correction and use the IR sextupoles for the $3 Q_{x}$ correction.

In this note, using the updated magnetic multipole field errors in the interaction regions, dynamic apertures are calculated in the tune range around the current pp working point. The effects on the dynamic apertures from nonlinear corrections, such as multipole field error correction in the IRs, second order chromaticity correction and horizontal third order resonance correction are evaluated. The sextupole components in the arc dipoles and the observed tune ripples are also considered. 


\section{Linear and nonlinear beam optics}

In this section, we will introduce the beam and optics parameters in the dynamic aperture calculations in next section. The schemes of the nonlinear corrections are also shortly reviewed.

To further increase the luminosity, we plan to increase the bunch intensity to $2.0 \times 10^{11}$, and reduce the $\beta^{*}$ down to $0.9 \mathrm{~m}$. There will be two collision points, IP6 and IP8. The $\beta^{*} \mathrm{~s}$ at the other IPs are $5 \mathrm{~m}$. The first order chromaticities are set to 2.0 before tracking, for both the 2-family and 8-family chromaticity correction schemes. Tab. 1 lists the beam and optics parameters in the following studies. The normal tunes shown in Tab. 1 are tunes without collision.

Table 1: The beam and optics parameters proposed for next pp run at current working point.

\begin{tabular}{lcc}
\hline \hline quantity & unit & value \\
\hline energy & $\mathrm{GeV}$ & 100 \\
normalized emittances $\epsilon_{n}, 95 \%$ & $\mathrm{~mm} . \mathrm{mrad}$ & 15 \\
single bunch intensity $N_{b}$ & & $2.0 \times 10^{11}$ \\
nominal Blue ring tunes $\left(Q_{x, 0}, Q_{y, 0}\right)$ in $2006 \mathrm{pp}$ run & $\ldots$ & $(28.695,29.685)$ \\
nominal Yellow ring tunes $\left(Q_{x, 0}, Q_{y, 0}\right)$ in 2006 pp run & $\ldots$ & $(28.685,29.695)$ \\
chromaticities $\left(Q_{x}^{\prime}, Q_{y}^{\prime}\right)$ & $\ldots$ & $(2.0,2.0)$ \\
$\beta_{x, y}^{*}$ at IP6 and IP8 & $\mathrm{m}$ & 0.9 \\
$\beta_{x, y}^{*}$ at IP10, IP12, IP2, IP4 & $\mathrm{m}$ & 5.0 \\
Total RF gap voltage & $\mathrm{kV}$ & 300 \\
\hline \hline
\end{tabular}

\subsection{IR multipole field errors and their corrections}

The field quality of the triplet quadrupoles and separation dipole magnets in the interaction regions greatly limits the performance of RHIC at the collision energy. To compensate their effect onto the dynamic aperture, local multipole corrector packages were installed at each side of interaction regions. However, at present, only the sextupole, skew sextupole and octupole correctors in IR6 and IR8 have power supplies connected. In the following simulation, only these correctors are used for local nonlinear correction. The correction strengths are derived with action-angle minimization by J. Wei [8]. As an example, Tab. 2 shows the chromaticity changes with and without IR multipole field errors.

Table 2: Chromaticities with and without IR multipole field errors.

\begin{tabular}{lc}
\hline \hline quantity & value \\
\hline without IR multipoles: & \\
$\left(Q_{x}^{\prime}, Q_{y}^{\prime}\right)$ & $(1.85,1.93)$ \\
$\left(Q_{x}^{\prime \prime}, Q_{y}^{\prime \prime}\right)$ & $(441,4998)$ \\
$\left(Q_{x}^{\prime \prime \prime}, Q_{y}^{\prime \prime \prime}\right)$ & $(211273,97407)$ \\
\hline with IR multipoles: & \\
$\left(Q_{x}^{\prime}, Q_{y}^{\prime}\right)$ & $(1.85,1.93)$ \\
$\left(Q_{x}^{\prime \prime}, Q_{y}^{\prime \prime}\right)$ & $(377,5020)$ \\
$\left(Q_{x}^{\prime \prime \prime}, Q_{y}^{\prime \prime \prime}\right)$ & $(2138484,71616)$ \\
\hline with IR multipoles and corrections: & \\
$\left(Q_{x}^{\prime}, Q_{y}^{\prime}\right)$ & $(1.85,1.93)$ \\
$\left(Q_{x}^{\prime \prime}, Q_{y}^{\prime \prime}\right)$ & $(433,4962)$ \\
$\left(Q_{x}^{\prime \prime \prime}, Q_{y}^{\prime \prime \prime}\right)$ & $(2112580,108581)$ \\
\hline \hline
\end{tabular}

\subsection{Sextupole components in the arc dipoles}

The sextupole components in the arc dipole affect the linear and nonlinear chromaticities. Based on the magnet measurements of $20 \%$ of the arc dipole magnets, at $100 \mathrm{GeV}$, the mean and rms sextupole components 
are $\left(k_{2} L\right)_{\text {mean }}=0.028 \mathrm{~m}^{-2}$ and $\left(k_{2} L\right)_{r m s}=0.0216 \mathrm{~m}^{-2}$, respectively.

In the following simulation, we insert a sextupole multipole in the middle of each arc dipole. Their strengths are generated randomly by a Gaussian distribution using $\left(k_{2} L\right)_{\text {mean }}$ and $\left(k_{2} L\right)_{r m s}$. As an example, at the working point $\left(Q_{x}, Q_{y}\right)=(28.685,29.695)$ with the 2 -family chromaticity correction scheme, Tab. 3 shows the chromaticities before and after installing sextupole components in arc dipoles. In this calculation, the above IR multipole errors and their corrections are included.

Table 3: Chromaticities with and without sextupole components in the arc dipoles.

\begin{tabular}{lc}
\hline \hline quantity & value \\
\hline without sextupole components: & \\
$\left(Q_{x}^{\prime}, Q_{y}^{\prime}\right)$ & $(1.85,1.94)$ \\
$\left(Q_{x}^{\prime \prime}, Q_{y}^{\prime \prime}\right)$ & $(2103,4327)$ \\
$\left(Q_{x}^{\prime \prime}, Q_{y}^{\prime \prime}\right)$ & $(1352037,-404868)$ \\
\hline with sextupole components but no $Q_{x, y}^{\prime}$ rematching: & \\
$\left(Q_{x}^{\prime}, Q_{y}^{\prime}\right)$ & $(10.07,-5.96)$ \\
$\left(Q_{x}^{\prime \prime}, Q_{y}^{\prime \prime}\right)$ & $(409,4773)$ \\
$\left(Q_{x}^{\prime \prime \prime}, Q_{y}^{\prime \prime \prime}\right)$ & $(2870488,-364383)$ \\
\hline with sextupole components but with $Q_{x, y}^{\prime}$ rematching : & \\
$\left(Q_{x}^{\prime}, Q_{y}^{\prime}\right)$ & $(1.85,1.94)$ \\
$\left(Q_{x}^{\prime \prime}, Q_{y}^{\prime \prime}\right)$ & $(463,4854)$ \\
$\left(Q_{x}^{\prime \prime \prime}, Q_{y}^{\prime \prime}\right)$ & $(2143975,86285)$ \\
\hline \hline
\end{tabular}

\subsection{Nonlinear chromaticity correction}

With the 2-family correction scheme, only the first order chromaticities can be controlled. With the 8-family correction scheme, we can minimize the nonlinear chromaticities while keeping the first order chromaticities. In our optimization with MADX [10], the second order chromaticities are corrected.

As an example, Tab. 4 shows the chromaticities at the working points $\left(Q_{x}, Q_{y}\right)=(28.685,29.695)$ with 2 and 8 family chromaticity correction schemes. In this calculation, the IR multipole field errors, their corrections, and sextupole components in arc dipoles are included.

Table 4: Chromaticities with 2 and 8 family corrections.

\begin{tabular}{|c|c|}
\hline quantity & value \\
\hline $\begin{array}{l}\text { with 2-family correction scheme: } \\
\left(\begin{array}{ll}Q_{x}^{\prime}, & Q_{y}^{\prime}\end{array}\right) \\
\left(Q_{x}^{\prime \prime}, \quad Q_{y}^{\prime \prime}\right) \\
\left(Q_{x}^{\prime \prime \prime}, \quad Q_{y}^{\prime \prime \prime}\right) \\
\end{array}$ & $\begin{array}{c}(1.85,1.93) \\
(557,5162) \\
(2166593,64671) \\
\end{array}$ \\
\hline 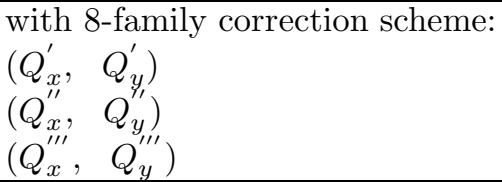 & $\begin{array}{c}(1.85,1.93) \\
(-78,41) \\
(-435147,-106114)\end{array}$ \\
\hline
\end{tabular}

\subsection{Third order resonance $3 Q_{x}$ correction}

As mentioned earlier, the horizontal third order resonance $3 Q_{x}$ is corrected with the 12 local sextupole correctors in the interaction regions. Note that so far only the 4 sextupole correctors in IR6 and IR 8 have power supplies.

As an example, for the 2-family chromaticity correction scheme, Tab. 5 shows the first order sextupole resonance driving terms and the chromaticities before and after the third order resonance correction. In this calculation, the IR multipole field errors, their corrections, and sextupole components in arc dipoles are included. 
Table 5: First order driving terms and chromaticities before $3 Q_{x}$ correction.

\begin{tabular}{lc}
\hline \hline quantity & value \\
\hline \hline before third order resonance correction & \\
Driving terms (real part, imaginary part): & $(5.17,-1.80)$ \\
$h_{21000}$ & $(4.70,3.66)$ \\
$h_{30000}$ & $(10.72,-4.48)$ \\
$h_{10110}$ & $(11.91,5.06)$ \\
$h_{10020}$ & $(11.12,1.40)$ \\
$h_{10200}$ & \\
\hline Chromaticities: & $(1.85,1.93)$ \\
$\left(\xi_{x}^{(1)}, \xi_{y}^{(1)}\right)$ & $(557,5162)$ \\
$\left(\xi_{x}^{(2)}, \xi_{y}^{(2)}\right)$ & $(2166414,64796)$ \\
$\left(\xi_{x}^{(3)}, \xi_{y}^{(3)}\right)$ & \\
\hline \hline after third order resonance correction & \\
Driving terms (real part, imaginary part): & $(8.38,-2.15)$ \\
$h_{21000}$ & $(0.10,-0.09)$ \\
$h_{30000}$ & $(4.51,-3.98)$ \\
$h_{10110}$ & $(9.44,3.33)$ \\
$h_{10020}$ & $(11.91,2.57)$ \\
$h_{10200}$ & $(1.85,1.93)$ \\
\hline Chromaticities: & $(526,4967)$ \\
$\left(\xi_{x}^{(1)}, \xi_{y}^{(1)}\right)$ & $(2098662,12914)$ \\
$\left(\xi_{x}^{(2)}, \xi_{y}^{(2)}\right)$ & \\
$\left(\xi_{x}^{(3)}, \xi_{y}^{(3)}\right)$ & \\
\hline \hline
\end{tabular}

\subsection{Tune ripples}

Tune ripples were observed in RHIC operation [9] and may reduce the beam dynamic aperture. Tab. 6 lists the tune ripples preliminarily measured with phase-locked loop tune meter. To check their effects on the dynamic aperture, in the following simulation, these tune ripples are artificially introduced by modulated quadrupoles at IP6. Each quadrupole represents one tune ripple. The tune ripples of one frequency in horizontal and vertical planes are of same amplitude and out of phase.

Table 6: Tune ripples observed in the RHIC [9].

\begin{tabular}{lcc}
\hline \hline $\begin{array}{l}\text { Frequency } \\
{[\mathrm{Hz}]}\end{array}$ & $\begin{array}{c}\text { One period } \\
{[\text { Turns }]}\end{array}$ & $\begin{array}{c}\text { Amplitude } \Delta Q_{\max } \\
{\left[10^{-5}\right]}\end{array}$ \\
\hline 60 & 1303.27 & 2.0 \\
120 & 651.64 & 3.0 \\
360 & 217.12 & 2.0 \\
720 & 108.61 & 2.0 \\
\hline \hline
\end{tabular}

\section{Tune footprint calculation}

In this section, the tune footprints are calculated below and above 0.7 in the tune space to check the available tune space to accommodate the beam-beam tune spread. The 4-D Tracking of on-momentum particle is performed with SixTrack [11]. The tunes are calculated with the $10^{3}$ turn-by-turn tracking data, using the modified Fast Fourier transformation (FFT) techniques. The 2-family chromaticity correction scheme is used here. The beam parameters are same as those shown in Tab. 1. 


\subsection{Tune space below 0.7}

Fig. 1 shows the tune footprints for the two working points $\left(Q_{x}, Q_{y}\right)=(28.685,29.695)$ and $\left(Q_{x}, Q_{y}\right)=$ $(28.695,29.685)$. From Fig. 1, with the beam-beam interactions on or off, the footprint with IR multipole correction is smaller than that without IR multipole correction.

With the beam-beam interactions, for working point $\left(Q_{x}, Q_{y}\right)=(28.685,29.695)$, the beam-beam interactions push the horizontal tune of the beam center to the horizontal third order resonance at $Q x=28.666$. And for the working point $\left(Q_{x}, Q_{y}\right)=(28.695,29.685)$, the beam-beam interactions push the vertical tune of the beam center to the vertical third order resonance at $Q_{y}=29.666$.

\subsection{Tune space above 0.7}

Limited by the resonances at 0.7 and 0.75 , and considering the beam-beam tune shift 0.02 , two working points $(28.735,29.745)$ and $(28.745,29.735)$ are used for tune footprint calculation above 0.7. Fig. 2 shows the tune footprints for these two working points. From Fig. 2, the IR multipole correction does help single particle's short-term stability above 0.7 , too.

Without the beam-beam interactions, for the working point $(28.735,29.745)$, the vertical tunes for large amplitude particles are close to the vertical fourth order resonance at $Q_{y}=29.75$. For the working point $(28.745,29.735)$, the horizontal tunes for large amplitude particles are close to the horizontal fourth order resonance at $Q_{x}=28.75$.

\section{Dynamic aperture calculation}

In this section, the dynamic apertures calculations are presented for the tune range around the current pp working point. The tracking is performed to $10^{5}$ turns. The 6-D tracking code SixTrack [11] is used. The initial relative energy deviation is 0.0007 . The beam-beam interactions are calculated with the weak-strong model. For each tune spot, the dynamic apertures are searched in five angles in the normalized coordinate frame. For comparison, in the following we only show the minimum dynamic apertures among these five angles. The dynamic apertures are given in unit of design rms beam size $\sigma_{0}$. To speed up the dynamic aperture search, a binary searching method is used.

\subsection{With and without IR multpole corrections}

As we mentioned earlier, we are able to locally correct the sextupole, quadrupole and skew sextupole multipole field errors in IR6 and IR8. Without beam-beam interactions, Fig. 3 and Fig. 4 show the dynamic apertures with and without IR multipole corrections, respectively. In both cases, the IR multiple errors are installed in all IRs. Comparing Fig. 3 and Fig. 4, the local IR multipole corrections do improve the dynamic apertures slightly along the diagonal in the tune space. In this calculation, the 2-family chromaticity correction scheme is used.

\subsection{With and without second order chromaticity correction}

With the beam-beam interactions on, Fig. 5 and Fig. 6 show the dynamic apertures in the tune scan with the 2-family correction scheme and with the 8-family correction scheme, i.e. without and with second order chromaticity corrections, respectively. In this calculation, the IR multipole errors and corrections are included.

Comparing Fig. 5 and Fig. 4, along the diagonal in the tune space, the beam-beam interactions at IP6 and IP8 decrease the dynamic apertures by about $1.0 \sigma_{0}$.

Comparing Fig. 6 and Fig. 5, with and without second order chromaticity correction, there is no significant change in the dynamic apertures along the diagonal in the tune space, except at the tune spot $(28.69,29.685)$.

\subsection{Effect of sextupole components in arc dipoles}

Fig. 7 and Fig. 8 show the dynamic apertures in the tune scan with sextuple components in arc dipoles with 2 and 8 sextupole families, respectively. Note that the non-collisional tunes and first order chromaticities are all re-matched to the design values before tracking begins.

Comparing Fig. 7 to Fig. 5, and Fig. 8 to Fig. 6, there is no significant change in the dynamic apertures with and without sextupole components in arc dipoles. 


\subsection{With and without third order resonance $3 Q_{x}$ correction}

The correction is done by compensating the $3 Q_{x}$ resonance's driving term $h_{30000}$ with the 12 local IR sextupole correctors, although now only 4 of these correctors have power supplied. In the correction, the first order chromaticities and the other first order sextupole resonance driving terms are unchanged.

Fig. 9 and Fig. 10 show the dynamic apertures calculated with the $3 Q_{x}$ correction with and without second order chromaticity correction, respectively. Comparing Fig. 9 to Fig. 7, and Fig. 10 to Fig. 9, above the diagonal in the tune space, the $3 Q_{x}$ correction does increase the dynamic apertures when the horizontal tune is approaching to $2 / 3$.

From Fig. 8 and Fig. 10, the $3 Q_{x}$ correction does not increase the dynamic apertures below the diagonal in the tune space since there the vertical third resonance $3 Q_{y}$ is the limitation. To correct the $3 Q_{y}$ resonance, the local IR skew sextupole correctors in the IRs should be powered.

\subsection{Effect of tune ripples}

The tune ripples are introduced by modulated quadrupoles at IP6. Fig. 11 and Fig. 12 show the dynamic apertures with tune ripples with 2 and 8 sextupole families. Comparing Fig. 11 to Fig. 7, and comparing Fig. 12 to Fig. 8, there is no clear dynamic aperture reduction due to the tune ripples in the $10^{5}$ turn DA trackings. Larger turn numbers may be needed to see their effect.

\subsection{Tune scan along the diagonal}

Here we calculate the dynamic apertures along the diagonal in the tune range from $Q_{x}=28.675$ to $Q_{x}=$ 28.745. The horizontal tune scan step is 0.005 . The vertical fractional tune is \pm 0.01 away from the horizontal fractional tune. In this study, the 2-family sextupole scheme is used. Fig. 13 shows the dynamic apertures in the tune scan.

From Fig. 13, when the non-collisional horizontal fractional tune is below 0.685 , the dynamic aperture drops quickly. And when the non-collisional horizontal fractional tune is near 0.72 , the dynamic aperture is reduced. Both of them can be explained by the total beam-beam tune spread of 0.02 . At $Q_{x}=0.685$, the beam-beam interactions push the colliding beam tune into horizontal third order resonance. At $Q_{x}=0.72$, the beam-beam interactions push the colliding beam tune into the horizontal tenth order resonance 0.7 .

\subsection{Tune scan above $\mathbf{0 . 7}$}

Fig. 14 shows the dynamic apertures in the tune scan above 0.7 in the tune space. The chromaticities are corrected with the 2-family sextupole scheme. From Fig. 14, the dynamic apertures reaches its maximum when $Q_{x}$ is near 0.745 . When the non-collisional horizontal fractional tune is near 0.72 , the dynamic aperture is reduced.

\section{Conclusion}

Detailed $10^{5}$-turn 6-D dynamic apertures have been calculated around the current RHIC polarization proton working point. The initial relative momentum deviation is 0.0007 . The model includes the updated IR multipole field errors and their corrections, the sextupole components in the arc dipoles, and tune ripples. The second order chromaticities and the horizontal $3 Q_{x}$ resonance can be effectively corrected before tracking. Based on the dynamic aperture calculations, we conclude:

- With the beam-beam interactions at IP6 and IP8, where we have $\beta^{*}=0.9 \mathrm{~m}$, and a bunch intensity $N_{b}=2.0 \times 10^{11}$, the dynamic aperture at good tune spots is about $6 \sigma_{0}$.

- The dynamic apertures of particles with the same momentum deviation 0.0007 are not affected by the nonlinear chromaticity correction.

- A correction of the $3 Q_{x}$ resonance driving term $h_{3000}$ leads to an increase in the dynamic aperture for tune spots above the diagonal.

- There is only a small effect of the inclusion of sextupole field errors in the arc dipoles on the dynamic aperture. 
- There is only a small effect of tune ripple on the dynamic aperture with only $10^{5}$ turns.

- Between 0.667 and 0.7 in the tune diagram, the tune space is tight to accommodate the beam-beam tune spread of 0.02 with $N_{b}=2.0 \times 10^{11}$.

- Between 0.7 and 0.75 in the tune diagram, the maximum dynamic aperture is reached when the noncollisional tunes are chosen to be near 0.745 . The non-collisional tunes should not be smaller than 0.72 .

\section{References}

[1] W. Fischer, Beam-beam and BTF, 2006 RHIC Accelerator Physics Experiments Workshop, November $2-3,2005$, BNL.

[2] S. Tepikian, private communications, 2005.

[3] Y. Luo, W. Fischer, S. Tepikian, D. Trbojevic, Online nonlinear chromaticity minimization, BNL C-AD AP-Note 263, Dec., 2006.

[4] Y. Luo, Nonlinear chromaticities and $3 Q_{x}$ resonance corrections, 2006 RHIC Accelerator Physics Experiments Workshop, November 2-3, 2005, BNL.

[5] Y. Luo, M. Bai, J. Bengtsson, W. Fischer, D. Trbojevic, Simulation of 3Qx Resonance Driving Term Measurement with AC Dipole Excitation, BNL C-AD AP Note-265, Jan., 2007.

[6] Y. Luo, J. Bentsson, W. Fischer, and D. Trbojevic, Simulation of proposed on-line third order resonance correction schemes, BNL C-AD AP Note-264, Dec., 2006.

[7] J. Bengtsson, The sextupole scheme for the Swiss Light Source(SLS): an analytical approach, SLS Note 9/97, March 7, 1997.

[8] J. Wei, in Proceedings of workshop on LHC interaction region correction systems, BNL, May 6-7,1999.

[9] P. Cameron, private communication, 2006.

[10] MAD code homepage, http://mad.home.cern.ch/mad/.

[11] SixTrack code homepage, http://frs.home.cern.ch/frs/. 


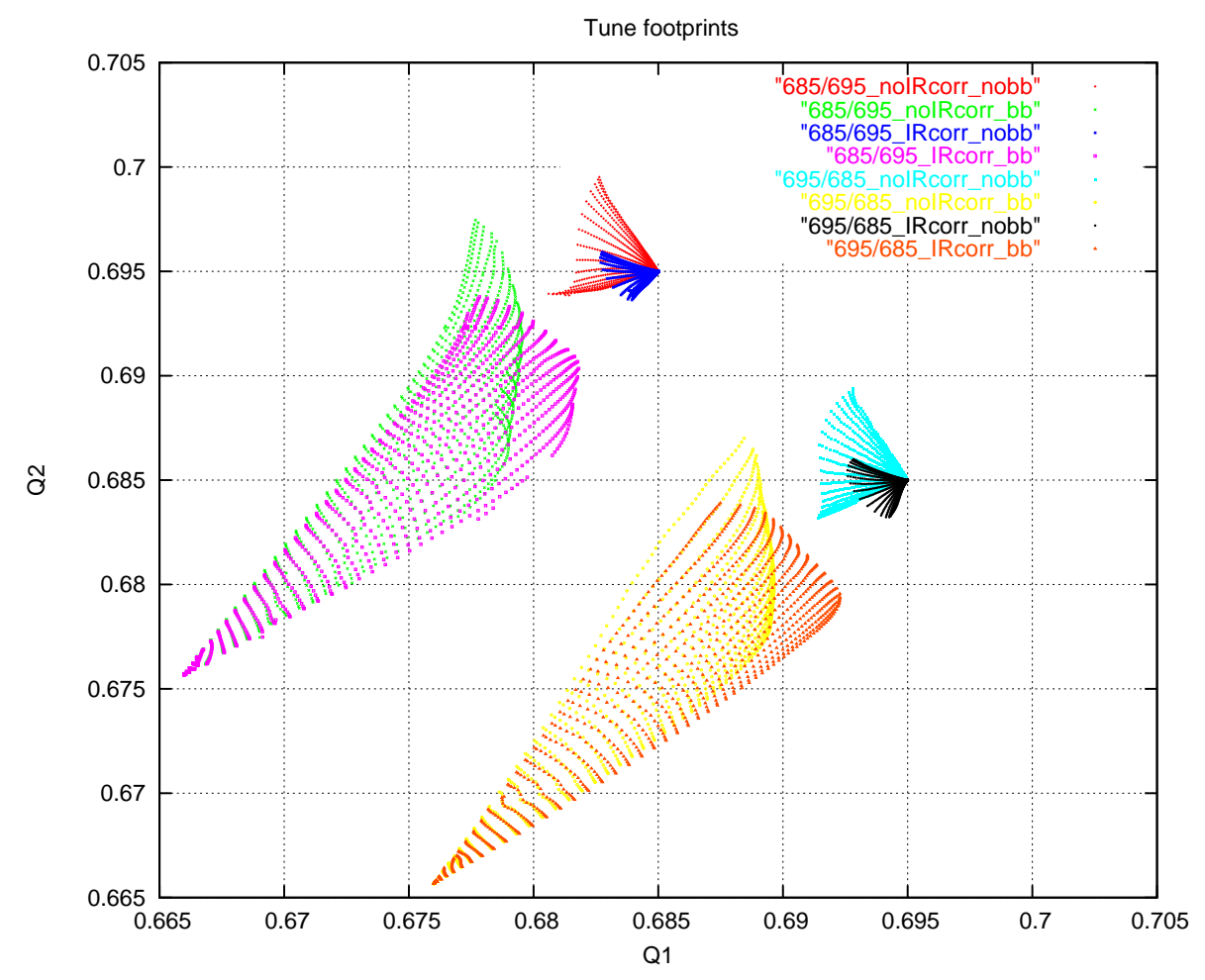

Figure 1: Below 0.7: The tune footprints with and without IR multipoles corrections for on-momentum particles up to $6 \sigma_{0}$.

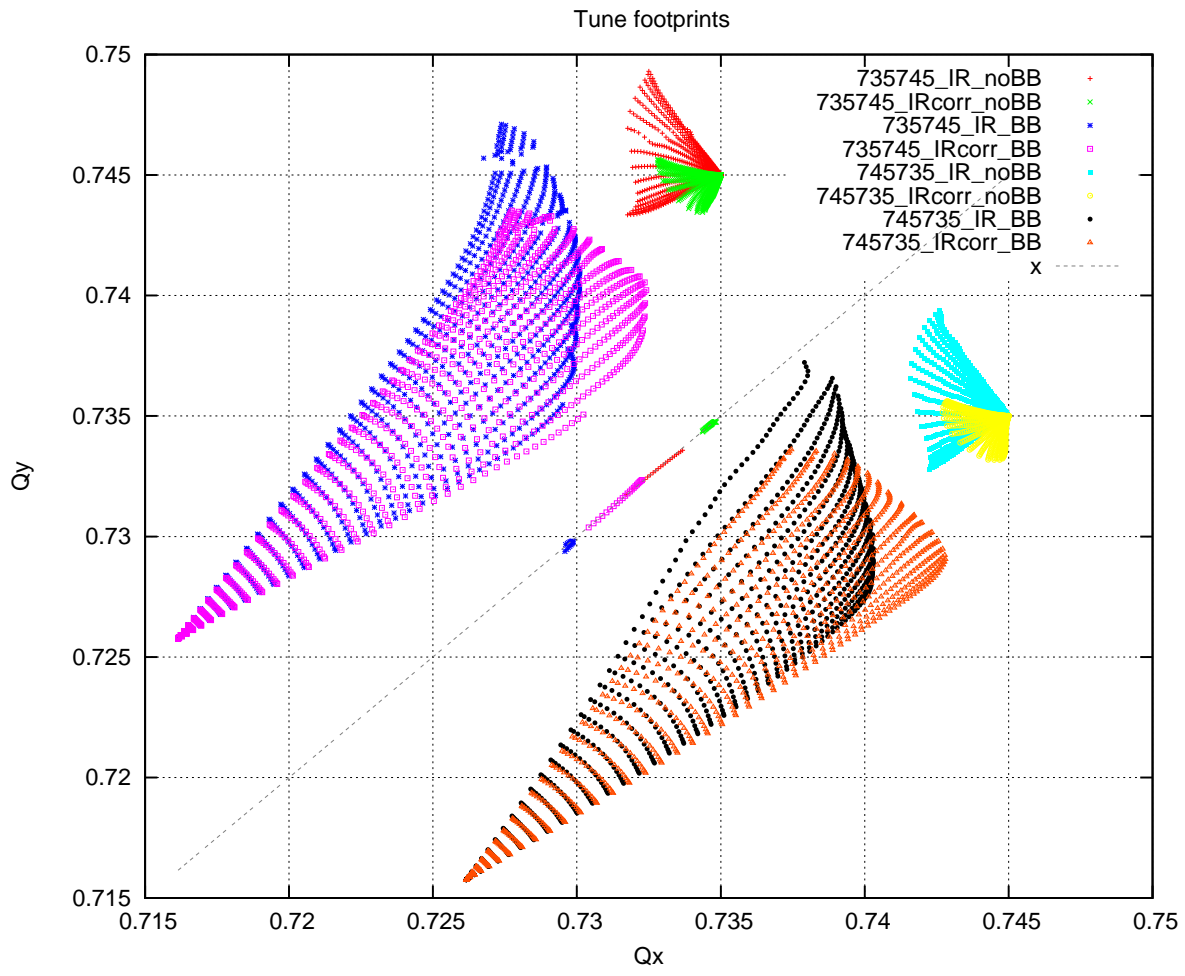

Figure 2: Above 0.7: The tune footprints with and without IR multipoles corrections for on-momentum particles up to $6 \sigma_{0}$. The tune spots on the diagonal are due to the failure in tune searching. 


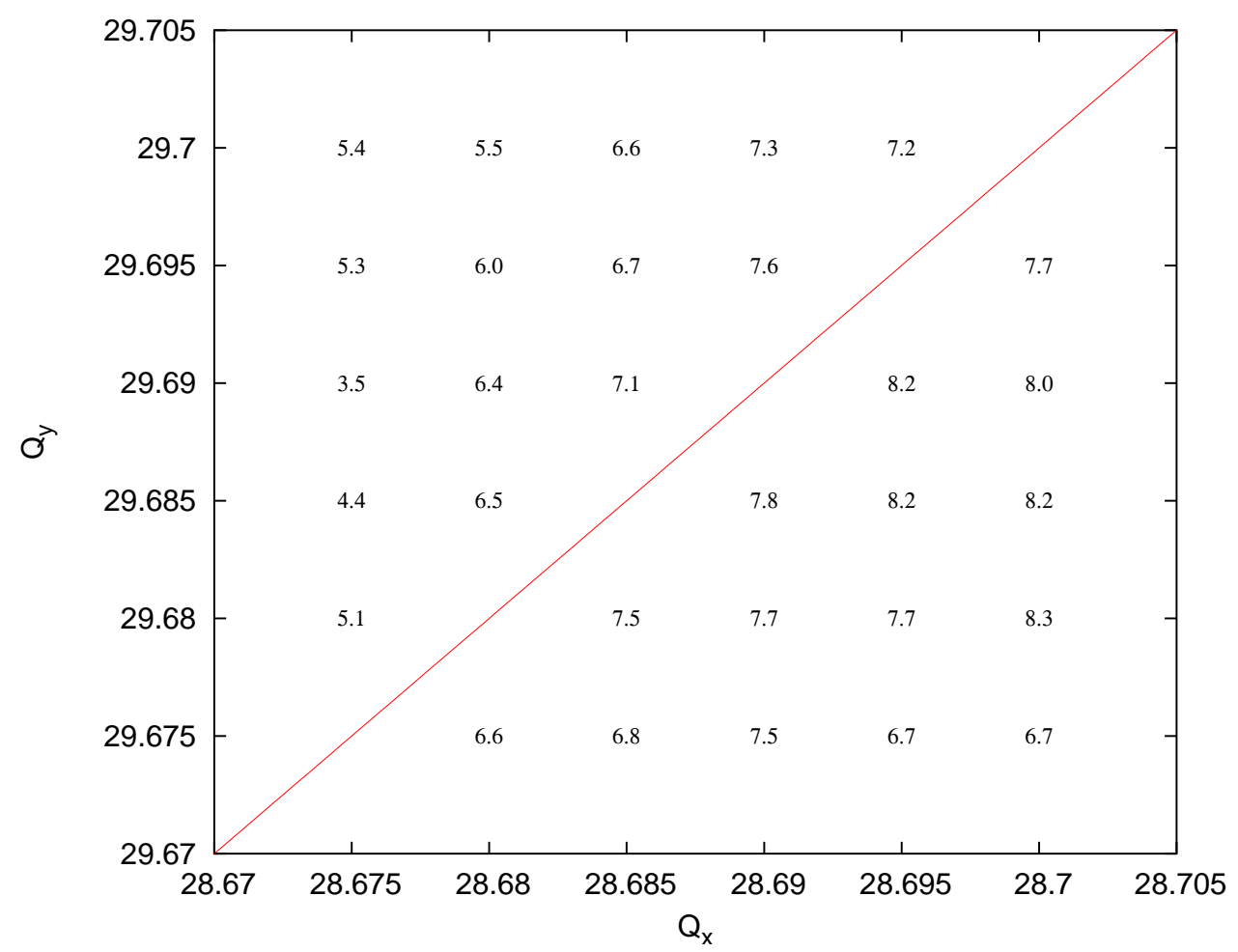

Figure 3: DAs in the tune scan under condition of $(\mathrm{IR}+2 \mathrm{fam})$. Beam-beam interactions are not included.

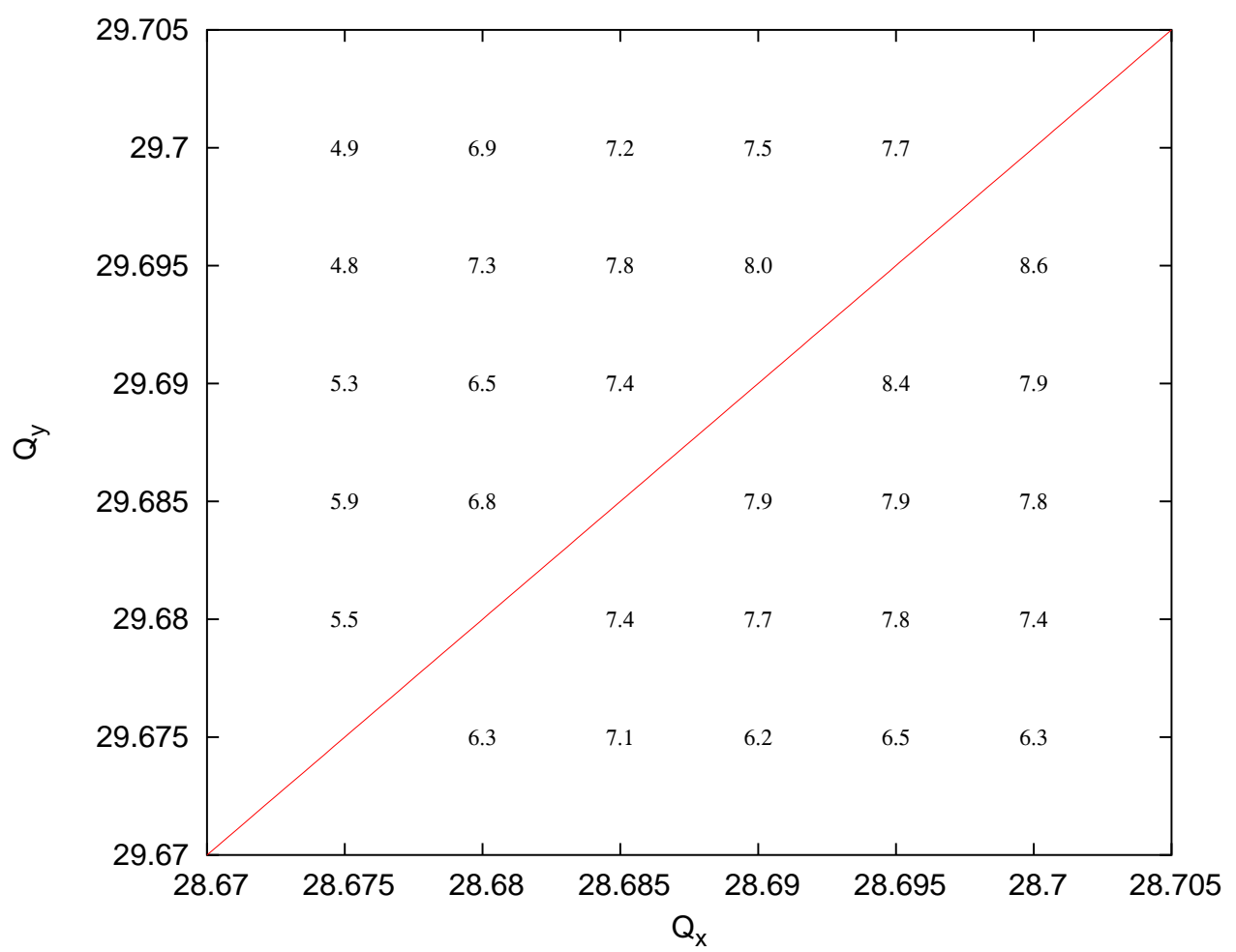

Figure 4: DAs in the tune scan under condition of (IRcorr +2 fam $)$. Beam-beam interactions are not included. 


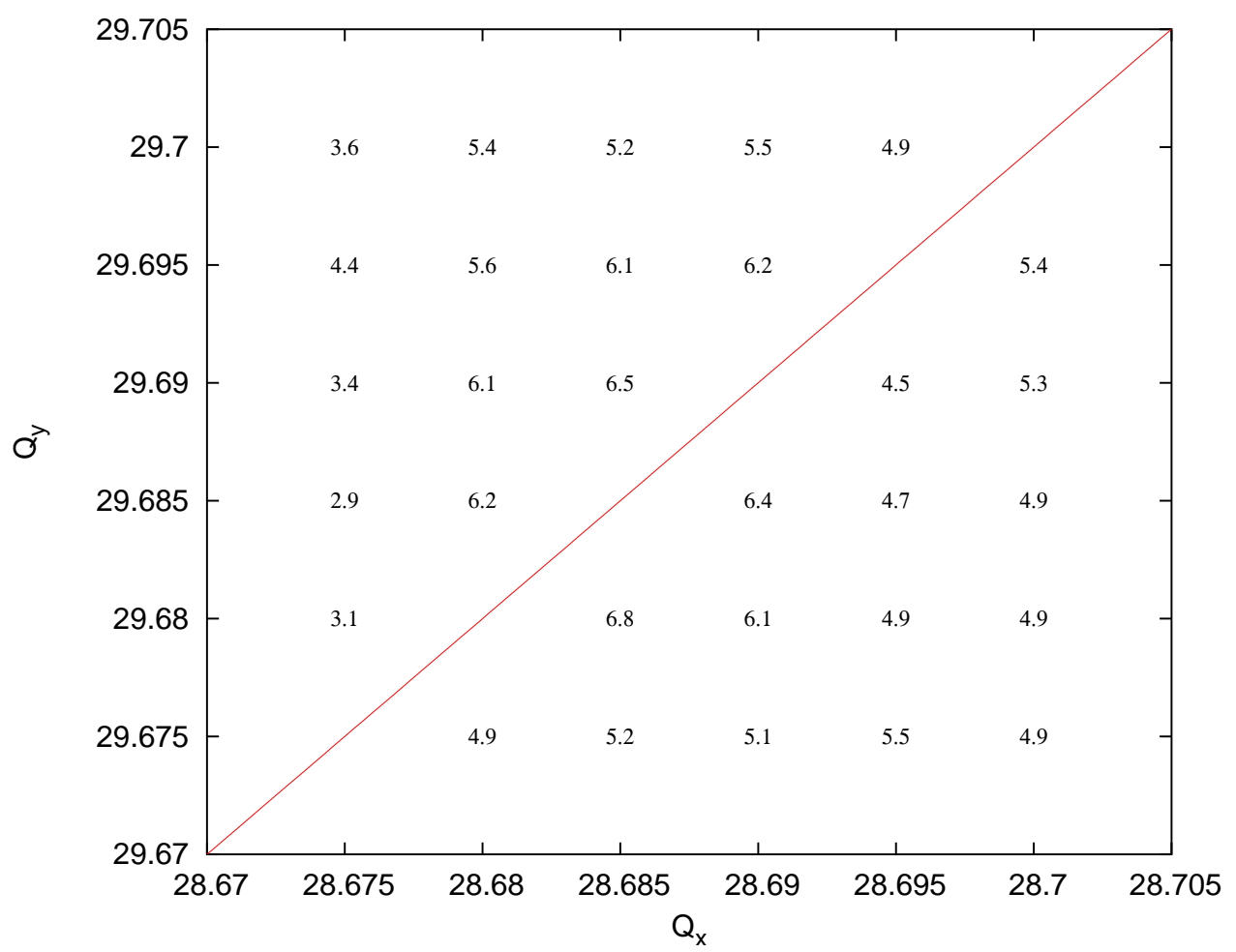

Figure 5: DAs in the tune scan under condition of (IRcorr + BB +2 fam $)$.

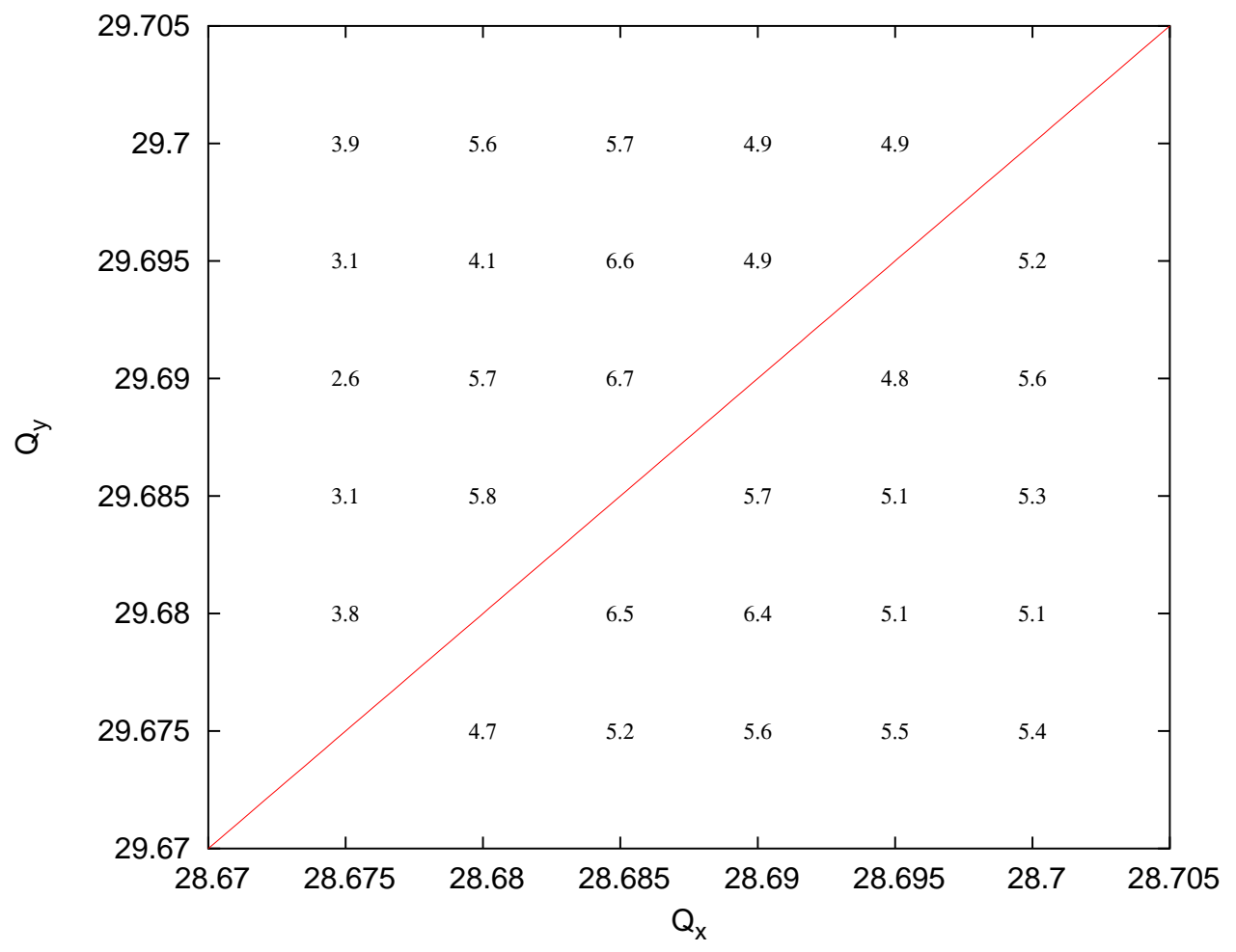

Figure 6: DAs in the tune scan under condition of (IRcorr $+\mathrm{BB}+8 \mathrm{fam})$. 


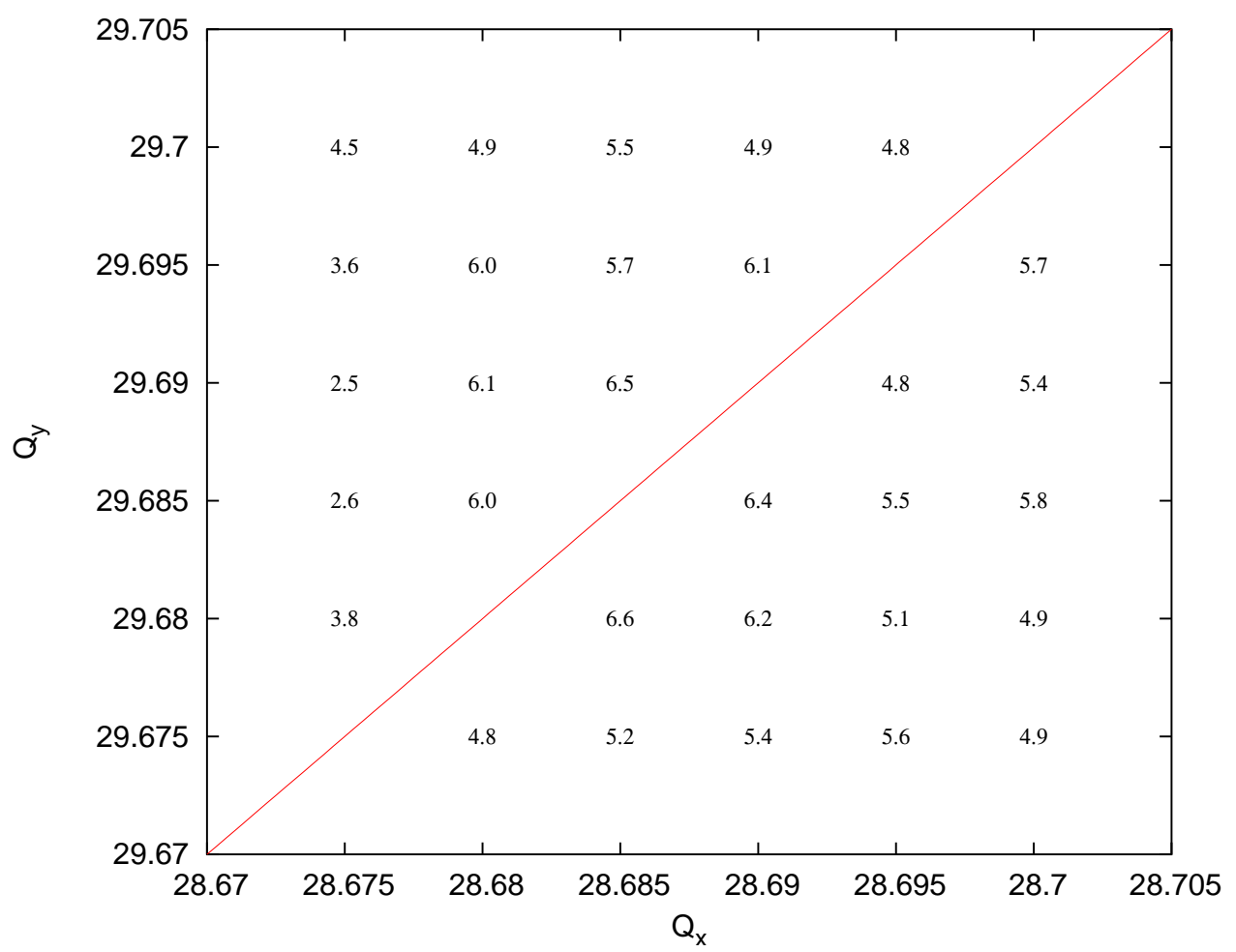

Figure 7: DAs in the tune scan under condition of (IRcorr + BB + b2 +2 fam ).

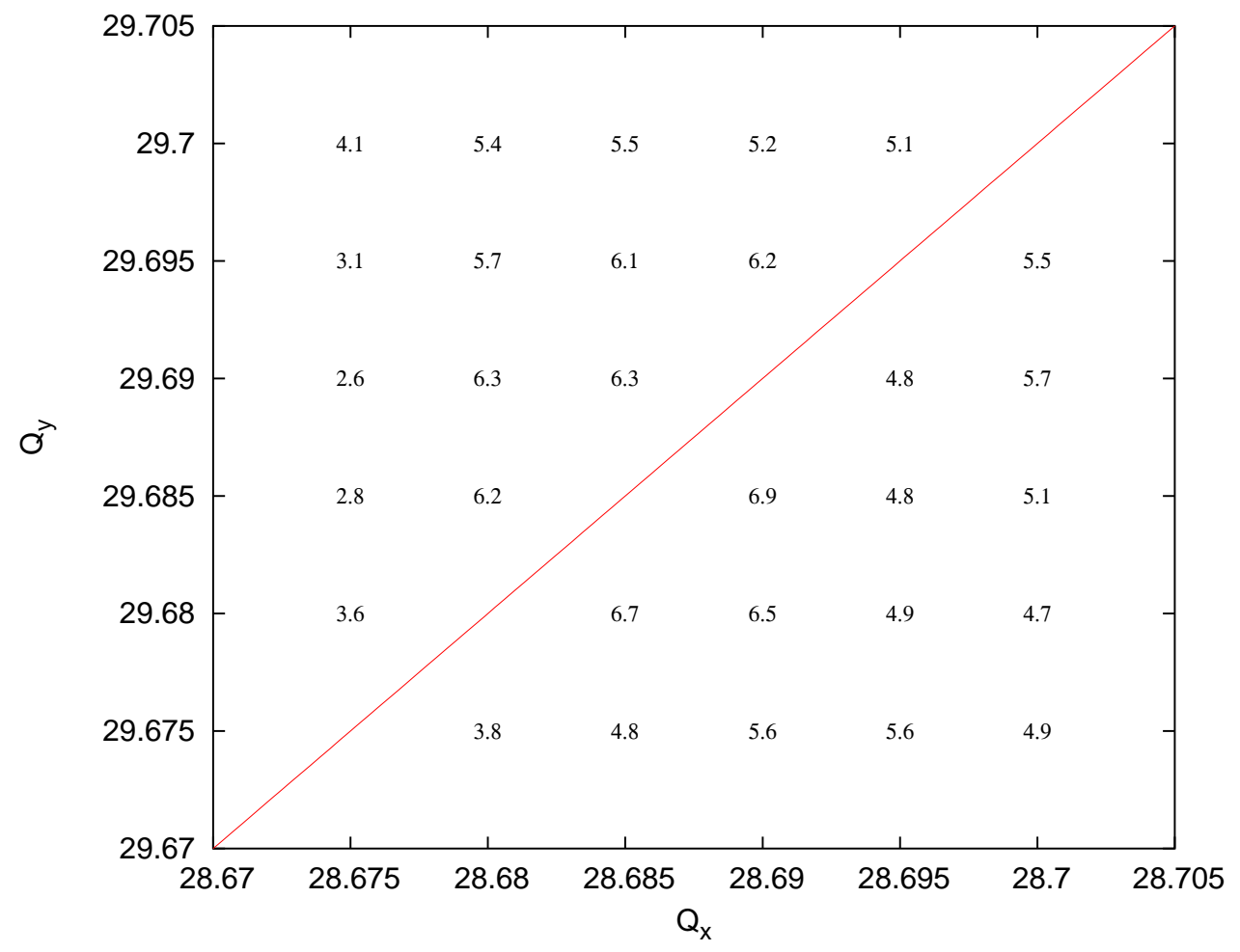

Figure 8: DAs in the tune scan under condition of (IRcorr + BB + b2 + 8fam ) 


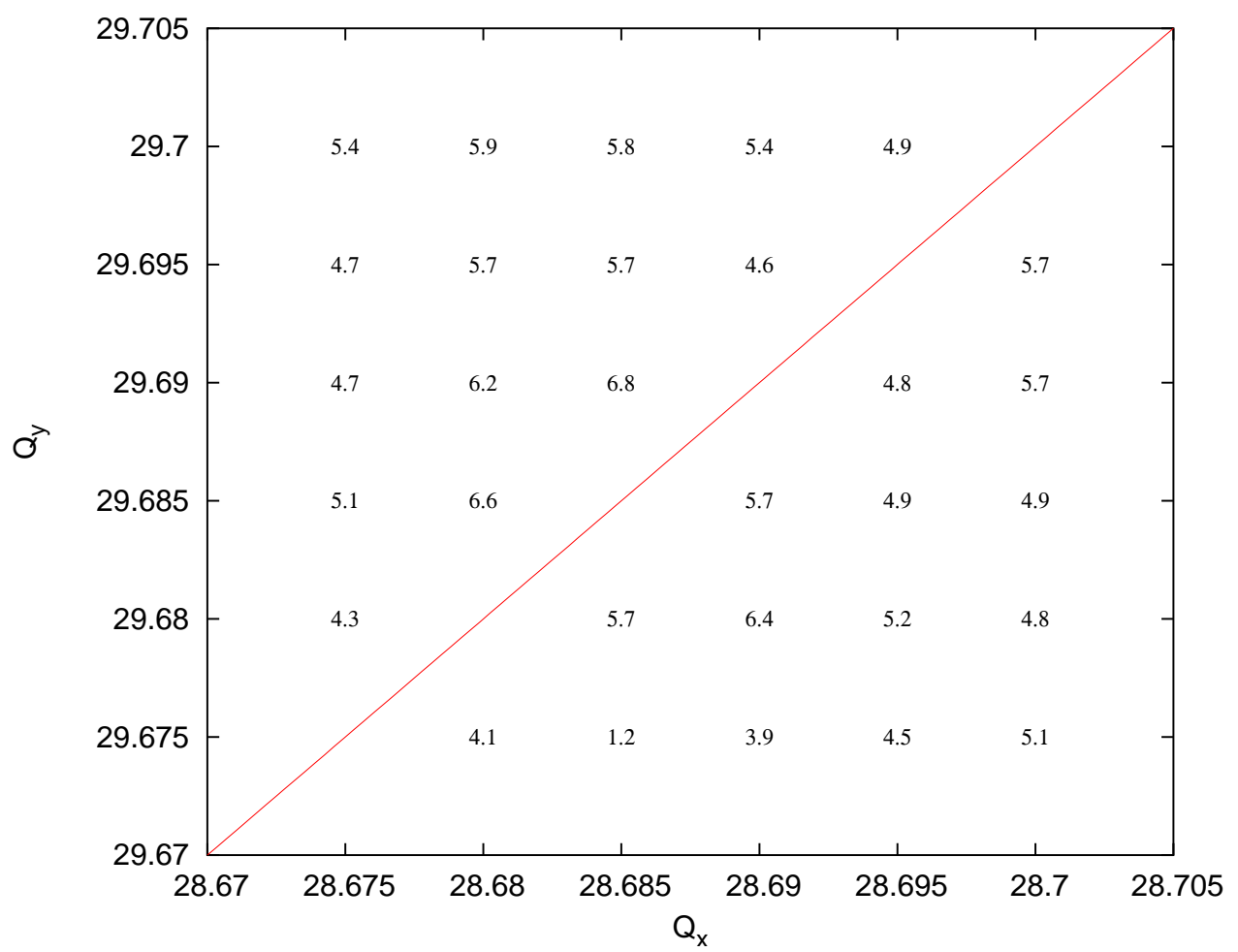

Figure 9: DAs in the tune scan under condition of (IRcorr $+\mathrm{BB}+\mathrm{b} 2+3 \mathrm{Qxcorr}+2 \mathrm{fam})$.

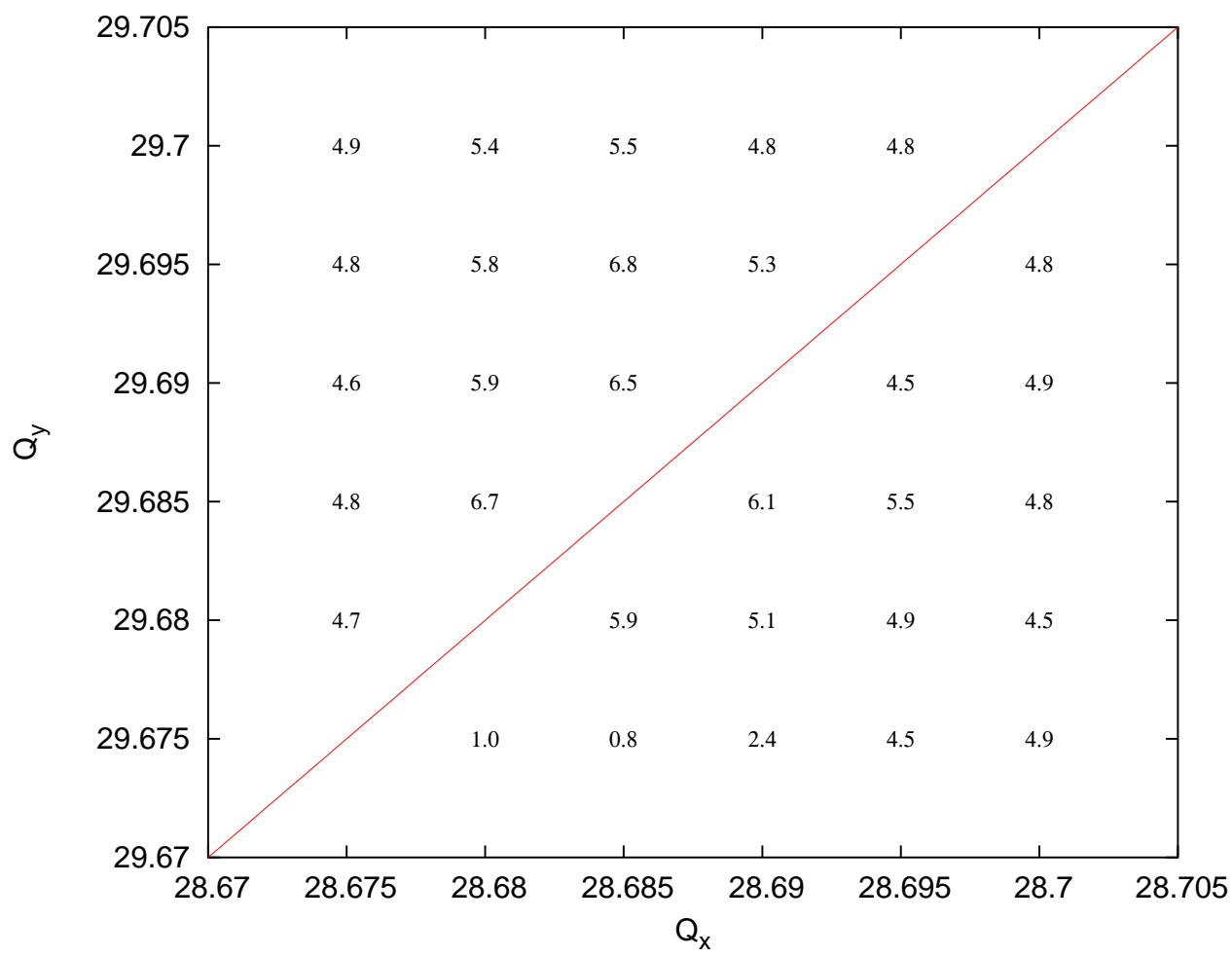

Figure 10: DAs in the tune scan under condition of (IRcorr $+\mathrm{BB}+\mathrm{b} 2+3 \mathrm{Qxcorr}+8$ fam $)$. 


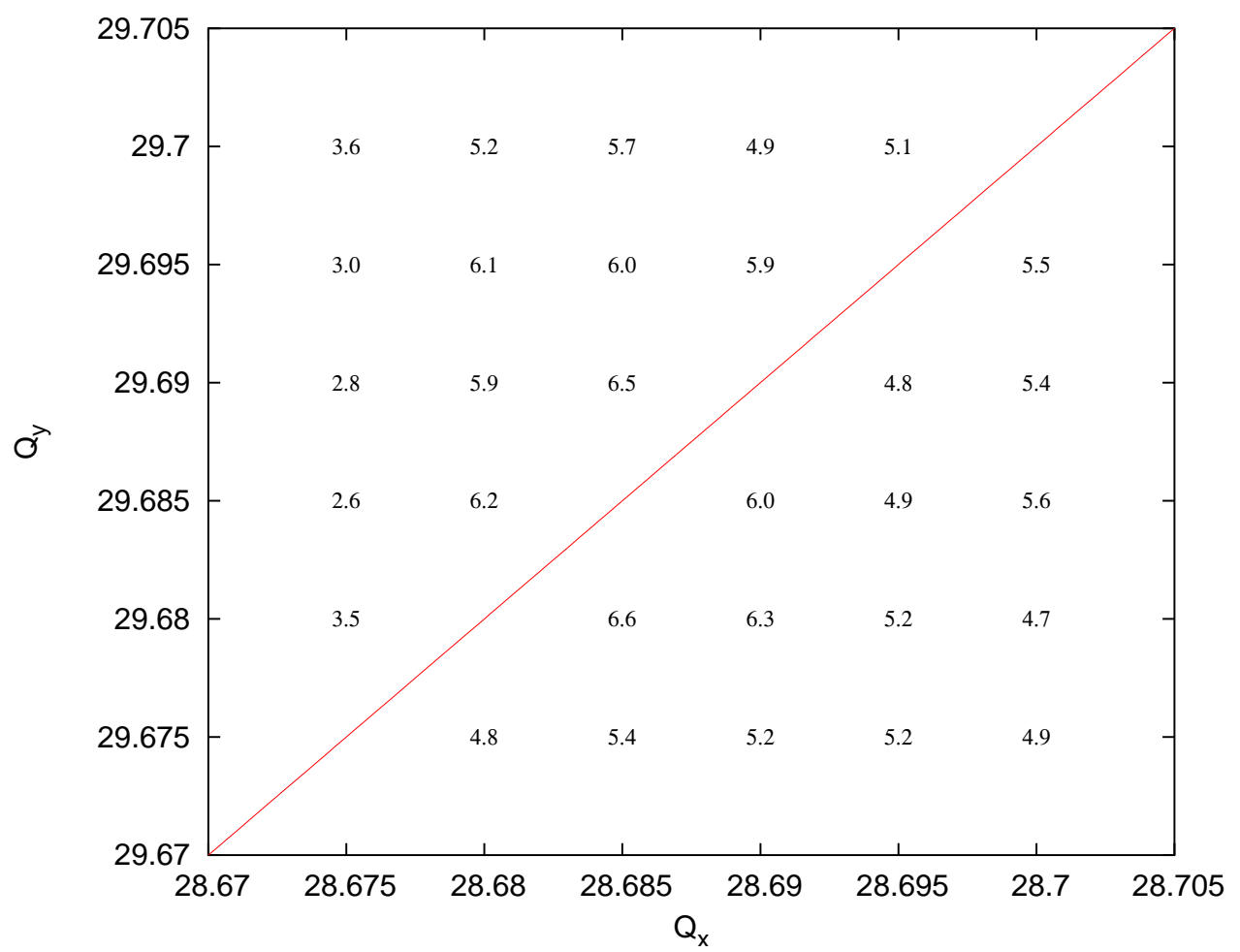

Figure 11: DAs in the tune scan under condition of (IRcorr $+\mathrm{BB}+\mathrm{b} 2+\operatorname{tr}+2 \mathrm{fam})$.

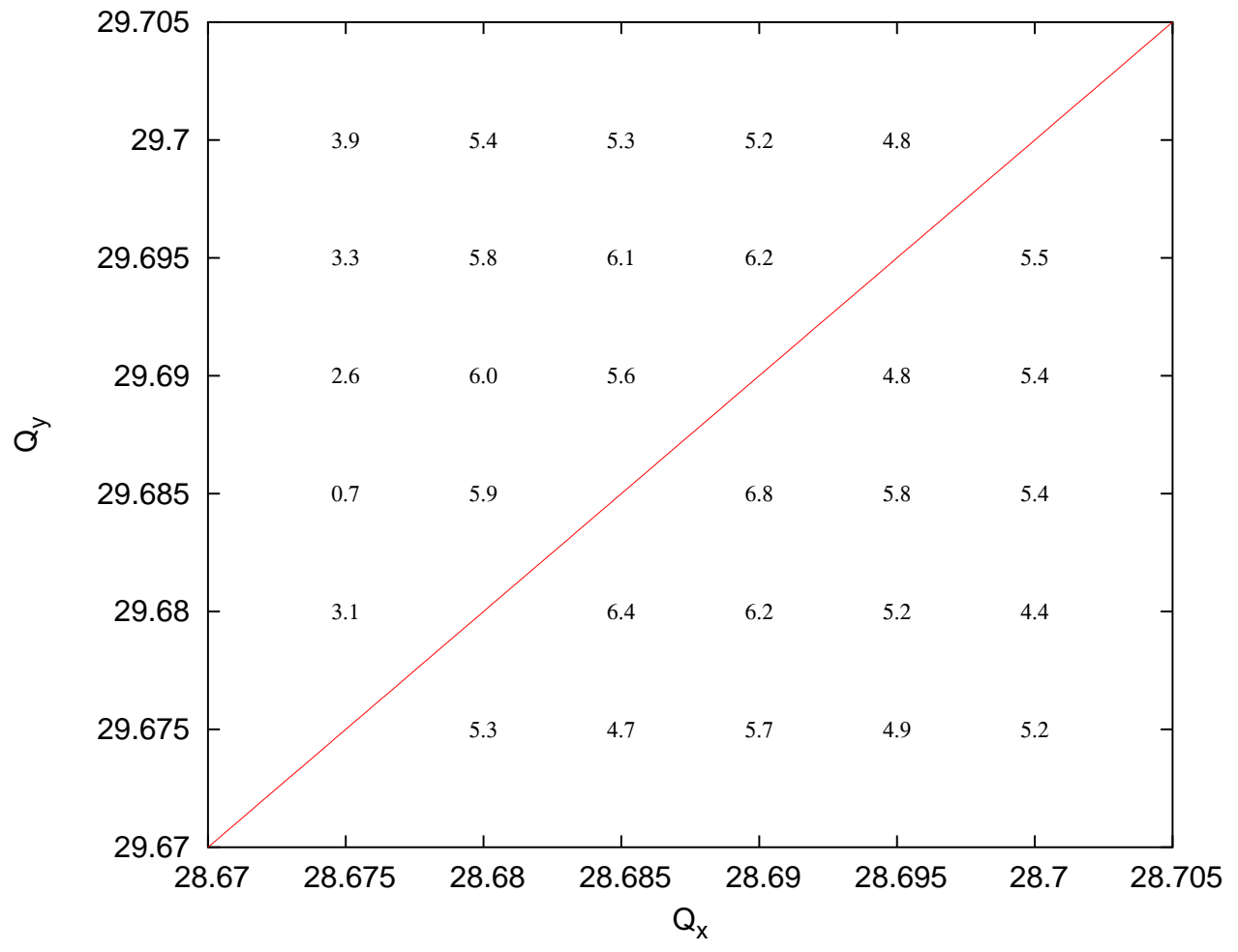

Figure 12: DAs in the tune scan under condition of (IRcorr $+\mathrm{BB}+\mathrm{b} 2+\operatorname{tr}+8 \mathrm{fam})$. 


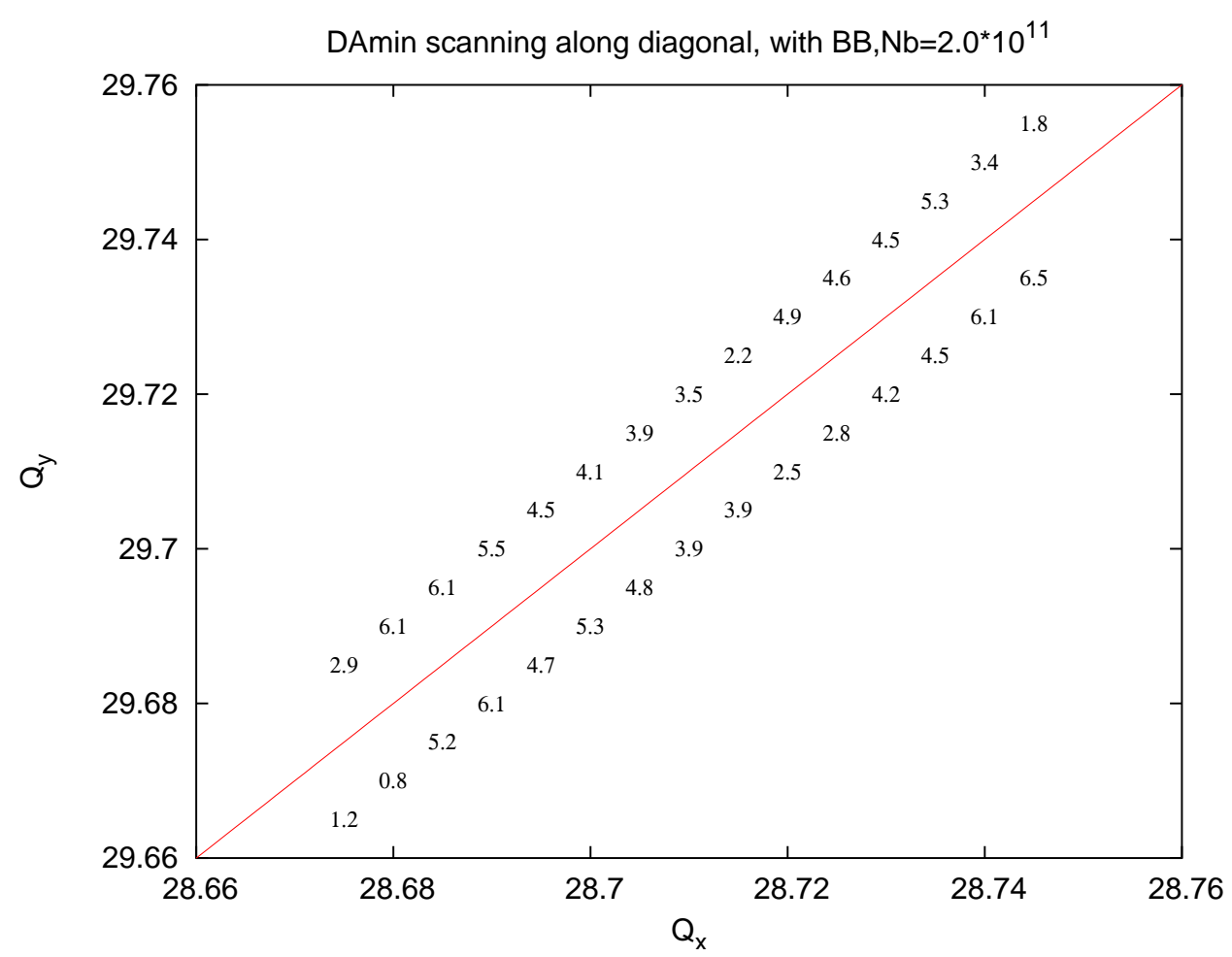

Figure 13: DAs in the tune scan along the diagonal line under condition of (IRcorr $+\mathrm{BB}+\mathrm{b} 2+2 \mathrm{fam})$.

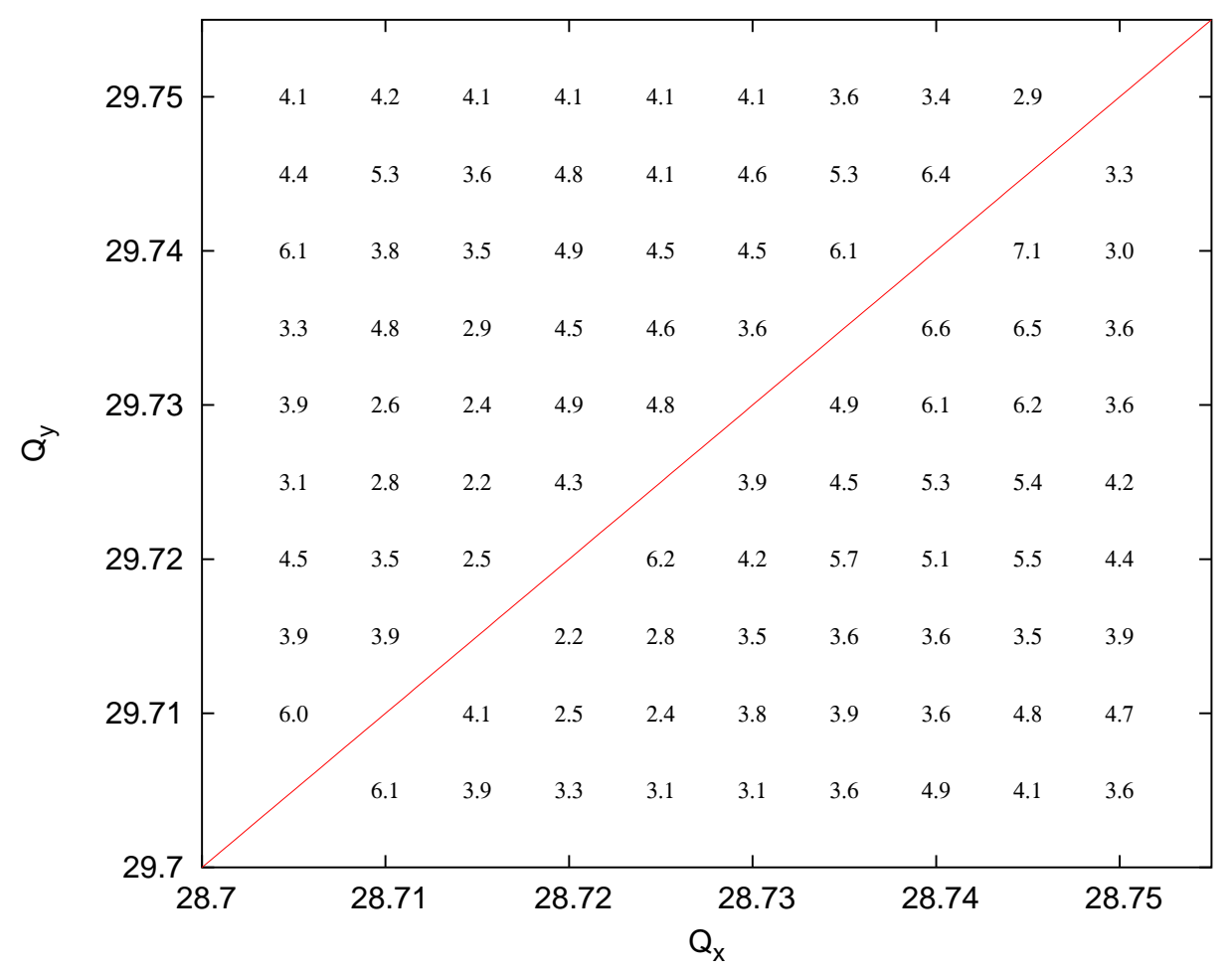

Figure 14: DAs in the tune scan above 0.7 under condition of (IRcorr $+\mathrm{BB}+\mathrm{b} 2+2 \mathrm{fam})$. 